\title{
Los impactos del turismo
}

\author{
Tomás franco Aliaga* y Óscar Garcia Guillén**
}

\begin{abstract}
RESUMEN SUMMARY
El turismo aparece, dentro de la economía española, como una actividad determinante desde los años sesenta. Sus efectos beneficiosos se han dejado sentir no sólo en la Balanza de Pagos o en el Producto Interior Bruto, sino que también han impactado favorablemente en el empleo y en la cultura. Sin embargo, esas repercusiones no han sido tan positivas en el medio ambiente, pues

nuestras costas sufren numerosos problemas urbanisticos, infraestucturales y de contaminación diversa.

Tourism appears, within the Spanish economy, as a determinant activity since the sixties. Its beneficial effects have showed not only on the Balance of Payments or the Gross Domestic Product, but also on employment and the culture. However, those effects have not been as positive for the environment since our coasts are suffering a lot from urbanism, infrastructure and pollution problems.
\end{abstract}

PALABRAS CLAVE Turismo, medio ambiente, contaminación, empleo, cultura.

\section{KEY WORDS}

Tourism, environment, pollution, employment, culture.

* Profesor Titular de la UNED

* Máster en Turismo y MBA. 


\section{INTRODUCCIÓN}

El turismo se comporta en España como una actividad estratégica en tres aspectos fundamentales: en el equilibrio de la Balanza de Pagos, en su aportación al Producto Interior Bruto (PIB) y en el impulso al empleo. Pero no acaban aquí sus efectos beneficiosos porque también ha dado al país una estampa internacional, decisiva «en una economía tan globalizada y mediatizada por la imagen y el mercado como la actual» (Vera, 1996: 337).

Sobre magnitudes de 1997 , el PIB turístico asciende a 8,4 billones de pesetas, superando en un $42 \%$ el de la construcción y triplicando el agrario y pesquero. Pero lo más interesante de las actividades turísticas está en su incidencia intersectorial y en su efecto multiplicador dentro de la economía, ya que por cada 100 pesetas consumidas en el subsector turístico se generan 171 en el conjunto del sistema.

De forma directa e indirecta las actividades turísticas dan trabajo al $10 \%$ de la población ocupada en España, aun cuando éste carezca de un buen nivel formativo y adolezca de un empleo poco estable. El impacto de las actividades turísticas sobre el medio ambiente es uno de los borrones de nuestro pasado y un reto para el futuro, que debe estar orientado hacia el ecoturismo. Finalmente se hacen en estas páginas algunas reflexiones sobre el impacto cultural que produce el turismo tanto en los países emisores como en los receptores, todos ellos anegados por esa inmensa avalancha turística que se mueve en las proximidades de los trópicos desde la década de los años 60 .

\section{EL IMPACTO ECONÓMICO}

En las últimas décadas el turismo se ha convertido en el hada madrina del desarrollo para numerosas regiones con escaso nivel de vida. Su influencia positiva ha empapado la casi totalidad de los subsectores económicos: desde los transportes aéreos a la hostelería pasando por la construcción, la artesanía o los espectáculos. Los gobiernos afectados, al margen de su ideologia, han abierto de par en par sus portones legales festejando la llegada masiva de las inversiones extranjeras en el subsector turístico, como ha sido el caso de Cuba (W.T.O., 1993).

La demanda turística tiene dos vertientes, la interior del propio país y la alimentada por corrientes llegadas de más allá de sus fronteras. La primera se comporta como un baremo del desarrollo y de la confianza de la gente en el sistema económico: cuando la situación es boyante la pobla- 
ción se desplaza, busca lugares de asueto y consume con largueza. Es el caso de España, donde el consumo turístico de los residentes en 1997 supera al de los no residentes, o propiamente turistas, en un $5,4 \%$ lo que representa casi medio billón de pesetas sobre un total de 8,6 billones.

El turismo se ha convertido en la actividad que mayores recursos moviliza a escala internacional y lleva camino de seguir en cabeza porque "crece más rápidamente que otras áreas de la economía" (loannides y Debbage, 1998:92). Cada vez se viaja más porque los niveles de renta son más elevados y las comunicaciones más rápidas y porque al aumentar el grado de formación se incrementa la curiosidad por otras culturas distintas con que enriquecer o afirmar la propia.

En nuestro ámbito occidental consideramos el trabajo como un recurso $y$, en consecuencia, también lo es el tiempo dedicado a él (El tiempo es oro). Sin embargo la inactividad no tiene un fin en sí misma, no aparece como la antítesis del esfuerzo remunerado sino que ha pasado a generar una industria dedicada al cultivo del tiempo libre. Paradójicamente, una sociedad condenada al trabajo según uno de sus mitos fundacionales ha hecho del ocio su recurso más cotizado, que canaliza a través del turismo. Esta doble visión del fenómeno como descanso rentable ha llevado a la Organización Mundial de Turismo (OMT) a definirlo como "una serie de actividades que realiza una persona al viajar y permanecer en un lugar fuera de su entorno habitual durante un periodo de tiempo inferior a un año y cuyo fin del viaje además de en sí mismo es realizar una actividad remunerada en el lugar objeto de la visita" (Joannides y Debbage, 1998: 36).

Del mismo modo que los occidentales y japoneses de finales del siglo $x x$ pasaron a disfrutar de los lugares de recreo reservados a los ricos de comienzos de la centuria, miles de millones de habitantes del resto del planeta, según vayan mejorando sus condiciones de vida, engrosarán la avalancha turística, integrada ya por 400 millones en 1994, que se transformarán en 650 millones en el año 2000 (Francescutti, 1997).

Para que el turismo tenga un impacto destacable en la economía de un país debe contar con varios factores. El primero hace referencia al factor de producción, que engloba tanto los recursos naturales como la abundancia de mano de obra y de capitales para atender la demanda turística. El segundo implica un desarrollo técnico avanzado para rentabilizar los citados recursos de producción. Conviene que los resortes utilizados sean propios a medio plazo, aun cuando en un primer momento haya de recurrirse a la importación de los mismos, porque una dependencia exterior continuada hace inviable la actividad. La aceptación social del hecho turístico es el tercer factor imprescindible, ya que un rechazo generalizado 
del mismo por parte de la población autóctona inhibirá la presencia del viajero. Finalmente, el lugar de destino precisa de una estabilidad social y económica reconocida porque los conflictos internos y las fluctuaciones monetarias al alza retraen o desvían la corriente turística hacia otras regiones con menores riesgos (Jiménez Moreno, 1997).

Antes de adentrarnos en el análisis de los impactos específicos del turismo en la economía española conviene señalar otra característica del mismo, lo que Cuadrado y Arranz denominan «efectos multiplicadores y carácter multisectorial del turismo» (1996: 205). Aunque no es fácil su cuantificación, prácticamente todas las actividades del sistema económico se ven afectadas en mayor o menor medida por el fenómeno difusor del turismo, lo que por otro lado demuestra la interdependencia de las partes dentro del citado sistema. Para ser más concretos, este mismo autor desglosa el origen sectorial del Valor añadido Bruto turístico atribuyendo el $3,7 \%$ a la agricultura, el $20,1 \%$ a la industria y energía, el $2,5 \%$ a la construcción y el $73,7 \%$ a los servicios. A la vista de estos datos podemos concluir que, aunque multisectorial, la economía turística gira fundamentalmente alrededor de los servicios y a gran distancia, de la industria.

Las actividades turísticas crean un inmenso caudal económico en forma de divisas y rentas que fluye desde las tierras altas de los países desarrollados hacia las bajas de los que están menos desarrollados, aunque ello no significa que los primeros no reciban cuantiosos beneficios desde otras zonas de desarrollo similar o intermedio. Para muchos países ubicados en los más bajos niveles, los ingresos por turismo han logrado aliviar, cuando no sanear, su balanza de pagos incidiendo de manera decisiva en el PIB y en el empleo. Pero si esto es así entre países también se repite el fenómeno en el interior de los mismos: el flujo se trasvasa de unas regiones a otras, aunque no siempre la corriente discurre de acuerdo con la ley de la gravedad. A veces el atractivo turístico está en las más desarrolladas, a donde acude la población en demanda de empleo. El resultado es una mayor despoblación de las regiones deprimidas y el recrudecimiento de los desequilibrios regionales. Veamos seguidamente con cierto detalle el impacto económico del turismo en esta última década sobre la Balanza de Pagos, el Producto Interior Bruto y el empleo en España.

\subsection{Impacto del turismo sobre la Balanza de Pagos y el PIB}

La economía española se enfrenta desde tiempos inmemoriales a una lacra estructural: el déficit de la Balanza de Pagos, situación típica de un país de desarrollo intermedio. En los años 60, época de la que arranca el 
fenómeno turístico, dicho déficit se financiaba por tres vías: ingresos por turismo, remesas de los emigrantes y entrada de capital foráneo a largo plazo. De las tres fórmulas, la turística fue la más importante por su volumen y por el impacto social que supuso para el país. Durante esa década los ingresos por turismo cubrieron como mínimo el $60 \%$ de la balanza comercial, llegando a superarla ampliamente en fechas posteriores (126\% en 1971). En algún momento esta afluencia de capitales por uno u otro conducto ha sido considerada como nuestro particular Plan Marshall que nos situó en la vía del desarrollo.

La crisis de los 70 trae nuevos saldos negativos en la Balanza de Pagos por cuenta corriente e incluso en la básica, y aunque los ingresos turísticos suavizan el problema "se reducen las reservas de divisas y se aumenta el endeudamiento exterior, con el fin de equilibrar la situación financiera con el exterior" (Bote, 1990: 61). El turismo representa ahora en nuestra economía el papel reservado en el siglo XVI a la lana merina, en el siglo XIX a los minerales $y$ en el $x x$ a los agrios. Se fundamenta en el llamado monocultivo del sol, considerado como un recurso inagotable para cuyo aprovechamiento no es preciso tomar medidas especiales. Grave error porque en todo momento ha de ser el turismo el que ayude a equilibrar la Balanza de Pagos puesto que consigue siempre mejores resultados en el mercado internacional que nuestros productos agrícolas e industriales.

Según el cuadro 1, la cobertura del déficit comercial a través de los ingresos turísticos refleja los altibajos de nuestras relaciones económicas con el exterior. Sin embargo, dichos vaivenes no se producen en el territorio de las actividades turísticas, en permanente incremento, sino en el de la economía española en su conjunto. Las tasas de cobertura tanto sobre las importaciones como sobre las exportaciones globales van decreciendo paulatinamente a la par que se fortalece el resto de nuestros subsectores económicos dentro del mercado internacional.

No es fácil singularizar para su cuantificación los ingresos y los gastos internacionales por turismo, que en 1997 ascendieron a un total de 3,9 billones y 662.000 millones de pesetas, respectivamente, lo que arroja un saldo positivo de casi 3,3 billones de pesetas, un 13,7\% más que en el año anterior. Las distintas partidas que conforman los ingresos son las siguientes: divisas ingresadas por los diversos servicios, otras partidas que corresponden a sueldos de españoles que trabajan en esta actividad, beneficios obtenidos en inversiones turísticas internacionales de bienes para consumo turístico interno, compra de servicios en el exterior, sueldos que se transfieren de los extranjeros que trabajan entre nosotros y beneficios que emigran por inversiones en el subsector. 
Cuadro 1. Impacto del turismo en la Balanza de Pagos y el PIB

\begin{tabular}{ccccc}
\hline Año & $\begin{array}{c}\text { \% sobre el déficit } \\
\text { comercial }\end{array}$ & $\begin{array}{c}\text { \% sobre } \\
\text { importaciones }\end{array}$ & $\begin{array}{c}\text { \% sobre } \\
\text { exportaciones }\end{array}$ & $\begin{array}{c}\text { \% sobre } \\
\text { el PIB }\end{array}$ \\
\hline 1980 & 55,0 & 21,0 & 35,0 & 9,10 \\
1985 & 139,0 & 29,0 & 36,0 & 9,30 \\
1990 & 65,6 & 23,4 & 35,9 & 9,34 \\
1997 & 146,0 & 21,9 & 25,8 & 10,81 \\
\hline
\end{tabular}

Fuente: Figuerola (1999b). Organización Mundial de Turismo (1999). Elaboración propia. Datos reales de 1997: Importaciones 17,9 billones; exportaciones 15,2 billones; déficit 2,7 billones; ingresos por turismo y viajes 3,9 billones; PIB 79,1 billones; PIB turístico 8,4 billones.

En el año 1997 el déficit de nuestra balanza de pagos fue de 2,7 billones de pesetas, ampliamente compensado por unos ingresos turísticos que alcanzaron la cifra récord de casi 4 billones de pesetas. $Y$ lo que es más interesante, a pesar de que los ingresos por turismo se incrementan año tras año (un $12,5 \%$ con respecto a 1996), sin embargo disminuye su índice de cobertura $(25,8 \%)$ porque los productos de otros subsectores de nuestra economía se consolidan en el exterior a un ritmo más intenso.

El consumo turístico para 1997 arroja un total de 8,6 billones de pesetas, cantidad que recoge el consumo tanto de los visitantes como de los residentes, este último algo superior al primero. Para hallar el PIB turístico multiplicamos la cifra de consumo por un factor de transformación $(0,9832)$, resultante de incorporar el IVA y otros impuestos indirectos (Figuerola, 1999b). El resultado es de 8,4 billones de pesetas, que alcanzan un $10,81 \%$ en el PIB nacional, porcentaje en continuo ascenso según nos muestra el cuadro 1.

Anteriormente hemos recalcado el efecto multiplicador y el carácter multisectorial que poseen las actividades turísticas hasta el punto de que casi ninguna zona de la economía escapa a su beneficiosa influencia. Eso nos lleva a valorar la producción turística global en 14,6 billones de pesetas, cifra que resulta de multiplicar el volumen de consumo turístico por un índice valorado en 1,71. Dicho de otra manera, se calcula que para producir 100 pesetas en el ámbito turístico hay que poner en circulación 171 en el conjunto de la economía (Figuerola, 1999a).

Al igual que la construcción, la actividad turística es un producto estructuralmente complejo que demanda y genera numerosos flujos en todas las direcciones, desde los agrarios con escaso valor añadido hasta los artesanales y financieros en los que la materia prima apenas cuenta. El im- 
pacto del turismo se deja sentir sobremanera en el comercio local y en la construcción. En el primer caso, el turista presiona sobre el consumo de bienes y servicios locales, que adquiere en un mercado ávido de beneficios rápidos porque su presencia se limita a un periodo corto de tiempo. Es el estío del hormiguero, que debe almacenar febrilmente para el largo invierno de la temporada baja. Hay casos, como ocurre con Ceuta y Melilla, y otrora con Andorra, en que los precios del mercado local son el objeto directo de la marea turística. Por una subida generalizada de los precios, esta situación de cierta desmesura comercial puede originar problemas entre los residentes, muchos de los cuales viven económicamente ajenos a la avalancha turística.

La influencia sobre la construcción ha tenido efectos inmediatos en la recalificación de suelos, aumento del precio de las viviendas, caos urbanístico en numerosos núcleos costeros, incremento de los niveles de contaminación, pérdida de las mejores tierras agrícolas para la construcción de infraestructuras y segundas residencias, etc. Mencionamos estos aspectos negativos porque entendemos que el impacto del turismo sobre la economía, aunque globalmente positivo, ha dejado unas secuelas amargas sobre el medio ambiente, algunas de difícil por no decir imposible solución, de lo que hablaremos más adelante.

\subsection{Impacto del turismo sobre el empleo}

Desde su aparición en la década de los años 60 el turismo ha mostrado una gran capacidad para la creación de empleo tanto en época de bonanza como de crisis económica. Es considerado como una actividad laboral intensiva, aun cuando muchas de sus prestaciones están siendo sustituidas por procesos mecanizados e informatizados. Pero el contacto directo con el público es todavía insustituible en numerosas fases de la citada actividad.

El empleo turístico se caracteriza por un elevado nivel de inestabilidad, bajos sueldos, condiciones de trabajo precarias y escasa cualificación. La precariedad es una consecuencia lógica de la fuerte estacionalidad o concentración de la mayor parte de la demanda turística en los meses de verano. Cuando el sol apenas calienta y hacen su aparición las primeras lluvias del otoño los turistas vuelven a sus tierras de origen. Se cierran numerosos establecimientos cercanos a la costa y la mano de obra va al paro. Ha concluido un ciclo estacional, que cada vez se intenta que sea más largo con ofertas a la tercera edad, congresos, reuniones, etc. 
A pesar del fuerte incremento $(88,5 \%)$ del numero de turistas en los últimos 16 años, pasando de 25,3 millones en 1982 a 47,7 en 1998, la población activa ocupada en el subsector ha aumentado sólo en un 10,2\%, con unos efectivos en torno a 1.300 .000 personas, de las que $« 770.000$ son empleos directos y 530.000 pueden estimarse en empleos inducidos, provocados por el multiplicador intersectorial de la actividad turística" (Figuerola, 1999b: 301). Esta cantidad supone en torno a un 10\% del total de la población ocupada, cuyas circunstancias sociolaborales representan uno de los más bajos peldaños del conjunto. Un gran número de trabajadores está contratado a tiempo parcial, mientras otros han de prolongar su jornada por encima de las ocho horas. Los salarios son reducidos y la cualificación escasa, de ahí que sea frecuente echar mano de trabajadores llegados del sector primario, de jóvenes sin completar su formación o de estudiantes universitarios que buscan novedades, vacaciones y algunos ingresos complementarios.

Al margen de estos aspectos negativos sobre la eventualidad, salarios y nivel de formación del empleo, hay que reconocer el cambio generacional que está sufriendo el subsector con abundancia de cuadros surgidos de las escuelas universitarias. Las nuevas modalidades de turismo invernal, de ocio para la tercera edad y de congresos están proporcionando una mayor estabilidad en el empleo aquí y en otros subsectores de la economía.

\section{IMPACTO DEL TURISMO SOBRE EL MEDIO AMBIENTE}

El turismo es una consecuencia del desarrollo económico y del estilo de vida. Detrás del fenómeno hay un planteamiento hedonista que da prioridad al consumo y al disfrute del entorno sin preocuparse por los ritmos de la naturaleza, por la renovación de los recursos y por la estética del medio. No pretendemos, sin embargo, dar la sensación de que las relaciones entre el medio ambiente y la actividad turística han sido un fracaso total. De hecho numerosas regiones han salido del subdesarrollo tras esta unión y se han agrandado las fronteras por el conocimiento de los pueblos. Pero el medio ambiente ha pagado un alto precio debido a nuestros impulsos depredadores, resumidos en esa fórmula tan en boga, pero medioambientalmente suicida, de usar y tirar.

En un primer momento pudo parecer que la preocupación por el medio tenía un carácter cultural o político limitado a grupúsculos ecologistas con ribetes exóticos. Años más tarde se ha convertido en un problema también para la economía porque el medio ambiente es ya un bien escaso no sólo por el consumo de materias primas (suelo, bosques, etc.) sino también 
por la pérdida de otros bienes más intangibles como el paisaje, las áreas de retiro y relajación, o el confort.

La fórmula propuesta por la OCDE (Organización de Cooperación y Desarrollo Económico) en 1975, quien contamina paga, ya no nos parece un principio de estricta justicia para restablecer la equidad sino una autorización encubierta. Si le damos la vuelta al precepto, éste abandona su tono conminatorio y se convierte en si puedes pagar, contamina. Es lo que de hecho ocurre debido a las multas irrisorias que se imponen, a la falta de controles públicos y a la insensibilidad de la Administración, la primera en incumplir los tratados internacionales sobre niveles de contaminación atmosférica.

Si es inevitable un punto de contaminación en algunos procesos económicos, también debe serlo la valoración de la misma para incorporar sus costes en los citados procesos. Es la llamada deseconomía externa a corregir con un impuesto equivalente a la diferencia entre el coste social y el privado (Díaz Álvarez, 1996). No es justo que dicho coste sea cero para la empresa mientras la sociedad invierte enormes sumas de dinero para descontaminar ríos, playas o bosques. A la corta esta medida es un mal menor, pero a la larga los individuos y la sociedad entera deben convencerse de que no hay mejor sistema sanitario que el preventivo, ya hablemos del medio ambiente o de nuestro organismo. Las medidas a tomar irían desde las netamente represivas a las que estimularan con subvenciones y ventajas fiscales una producción limpia y respetuosa con el medio (Franco Sala, 1995).

El impacto del turismo sobre el medio ambiente ofrece una gama variadísima de tropelías y de enloquecidos palos de ciego: edificios gigantescos a pie de playa, chalets colgados de los riscos, basuras repartidas sin control, aguas contaminadas, carreteras intransitables, ruido hasta el amanecer, pérdida de tierras fértiles, degradación de monumentos históricos, etc. Toda esta serie de desmanes aparece bien en la fase de iniciación de las actividades turísticas o en la de explotación de las mismas. El proceso de puesta en marcha conlleva un impacto estructural sobre el medio que se traduce en la construcción de carreteras, conducciones de agua y edificaciones. La siguiente fase de explotación turística incide sobre el consumo de recursos (agua), acumulación de vertidos (basuras) y contaminación atmosférica (automóviles, calefacciones e incendios).

\subsection{Dificultades para la conservación del medio ambiente}

Si la conservación del medio ambiente es un bien común del que todos nos beneficiamos y su deterioro conlleva riesgos de difícil control, parece 
lógico hallar un rápido consenso sobre qué hacer al respecto. Sin embargo, no es así porque el interés a corto plazo, y no la lógica, domina nuestras actuaciones. Más aún, hasta fechas recientes el desarrollo económico parecía reñido con la conservación del medio en las áreas deprimidas. Tal ha sido el caso de numerosas islas del Caribe o el de las Baleares (Morey, 1991) y las Canarias en la década de los años 70, época en que un turismo descontrolado imponía sus exigencias. Sobrevivir, mejorar el nivel de vida y en muchos casos especular eran actividades prioritarias sobre la protección del medio.

Con harta frecuencia muchas de las lacras medioambientales que produce el turismo se generan en los paises de origen, donde la propaganda crea y orienta las expectativas de los futuros visitantes. Los touroperadores venden un producto que pocas veces coincide con la realidad y menos aún con los intereses de las zonas de acogida. Según esto el turismo se presenta como un fenómeno tridimensional: por un lado está la imagen preconcebida del viajero en su lugar de origen, casi siempre distorsionada; por otro aparece la realidad con la que ese viajero se encuentra, poco o nada coincidente con su esquema mental, y por último están las expectativas, ya sean económicas o de comportamiento, que su llegada suscita entre los paisanos.

Incidiendo más en alguno de tales aspectos, la imagen que circula de un destino turístico en los países de origen condiciona o incluso determina el segmento de mercado o tipo de clientela. Ello no significa que sea fácil seleccionarlo porque se trabaja con un amplio abanico de naciones, a veces pertenecientes a culturas distintas.

No es el caso de los países europeos pues, como ha demostrado la organización ESOMAR (The European Society for Opinion and Market Research) (1998), éstos poseen características muy similares en el plano turístico, de las que también participa el resto de las sociedades industrializadas.

En los lugares de destino el turista es percibido como una fuente de divisas, sin que en ningún momento se lleve a cabo un balance de pérdidas y ganancias globales, incluido el medio ambiente. Algo está cambiando últimamente esta postura como lo prueba la preocupación por restringir el número de visitantes en algunas zonas, llegándose incluso a proponer una tasa turística.

Las dificultades para valorar el impacto turístico en el medio ambiente son obvias porque no es fácil prever el nivel de saturación, ya sea en el plano físico (infraestructuras, consumo de agua, sanidad, etc.), en el medioambiental (contaminación, paisaje, ruidos) o en el psicológico (grado 
de aceptación y respeto por ambas partes). Como ya se ha escrito reiteradamente, los intereses tanto de los visitantes como de los paises receptores son con frecuencia contrapuestos (Pearce, 1989).

En la Evaluación del Impacto Ambiental (ElA) entran en juego numerosos aspectos que permiten restañar las heridas abiertas y evitar los males futuros regulando por ley el control de los recursos hidráulicos y mineros, protegiendo las tierras más fértiles, el ecosistema o el patrimonio cultural (Ruesca y Durán, 1995). Como fuente de información y método de análisis la ElA es de gran interés para la toma de decisiones porque ahorra costes en la ejecución de proyectos y permite el seguimiento de los mismos. Desde mediados de 1995 existe un Reglamento de la UE (Unión Europea) que faculta a las empresas la adhesión a un sistema comunitario de gestión y de auditoría ambiental.

\subsection{El ecoturismo como alternativa}

El ecoturismo es un nuevo concepto que introduce la actividad turística dentro de la ecología, una rama de la Biología que estudia ias relaciones entre el hombre y su hábitat, y los efectos de la actuación del hombre con el fin de evitar que se ponga en peligro la permanencia de la vida sobre la Tierra (Blázquez, 1997).

Es una forma respetuosa con el medio ambiente que va ganando cada vez más adeptos en la medida en que aquél se degrada en las ciudades y en las zonas tradicionales de veraneo. Es el modelo turístico del futuro resumido en la fórmula gestión sostenible por la que un lugar turístico limitaría el número de visitantes cuando corriera el riesgo de preservarse su conservación. Ya se está poniendo en práctica en algunos parques naturales (Doñana), monumentos prehistóricos (Cuevas de Altamira) y bosques singulares (Hayedo de Montejo de la Sierra), entre otros puntos de interés turistico.

Hasta fechas recientes tanto los organismos públicos como las empresas privadas del ámbito turístico han considerado los recursos del medio como inagotables y por tanto carentes de consideración. Los resultados están a la vista: masificación costera, playas insalubres y contaminación de muy variada índole, fenómenos todos ellos en las antípodas del ecoturismo. Éste se está imponiendo como una poderosa corriente flanqueada por una filosofía o modo de entender el medio que nos rodea desde nuevos criterios estéticos y hedonistas, y por un estado de necesidad a la vista de los desequilibrios y aun desastres que conlleva tanto descontrol y 
abuso. El medio ambiente ya no es un bien libre sino económico por frágil y escaso, y su consumo ha de realizarse en pequeñas y equilibradas dosis para que permanezca intacto (Vera, 1992).

El movimiento turístico se ha convertido en un imponente oleaje por encima de los 650 millones de personas, cuya repercusión sobre el medio ambiente puede ser catastrófica (ya lo ha sido en numerosos puntos de nuestro litoral) si no se ataja desde los planteamientos del ecoturismo. La gestión sostenible de la que hablábamos con anterioridad recibe también, para este caso concreto, la denominación de turismo sostenible, cuyo fin primordial no es la búsqueda del beneficio económico sino la conservación del medio implicando a los habitantes de la zona. Sólo con su plena integración en el proyecto será posible mantener el equilibrio entre naturaleza, disfrute y realidad social, la armonía entre los agentes naturales y sociales que Barkin (1996) denomina desarrollo dual.

Entre los numerosos beneficios que fluyen de un turismo sostenible, según la Organización Mundial de Turismo (1993), destacamos los siguientes de carácter global: a) mantenimiento del equilibrio del medio; b) mejora de la calidad de vida de la comunidad receptora y c) oferta a los visitantes de una experiencia de elevado nivel ecológico. Para la OMT cualquier actuación debe estar guiada por «principios éticos de respeto a la cultura y al medio ambiente de la zona receptora, a su economía y forma tradicional de vida" (1993: 59).

\section{EL IMPACTO CULTURAL}

Hacia finales de los años 60 Defert catalogó los recursos turísticos en cuatro grandes grupos vinculados con las aguas terrestres, marinas, termales, etc. (hidrom), con el relieve y la vegetación (fitom), con los restos arqueológicos, museos y monumentos (litom), y con el folclore y la artesanía (antropom). El turismo cultural se alimenta sobre todo de los dos últimos y aparece como un contrapunto a la hegemonia tradicional del combinado sol y playa (Vera, 1997).

El fenómeno turístico es una expresión más de la curiosidad humana, que se pone en movimiento cuando un grupo social alcanza un nivel económico desahogado. Podríamos añadir que actualmente ya es una forma de terapia colectiva y de salida de escape al estrés laboral, lo que no obsta para que las vacaciones tengan ocasionalmente un marcado tinte de integración social con los lugares de destino, donde se descubren valores olvidados por las sociedades industrializadas. En este sentido Europa vive 
cada año un vasto movimiento migratorio desde las áreas norteñas hacia las zonas del Mediterráneo buscando sol y playa, en primer lugar, pero también gastronomía, folclore y cultura.

El impacto turístico sobre los lugares de destino varía en función de las características sociales de los usuarios, como son el estilo de vida, el nivel cultural y los recursos económicos disponibles (Cooper, 1993). De ello se deduce que las culturas muy tradicionales se ven más afectadas por presencias foráneas que aquellas otras caracterizadas por su cosmopolitismo, como es el caso de nuestras grandes ciudades y zonas costeras frente a las regiones rurales del interior.

Son numerosos los factores socioculturales que han impulsado esta modalidad más refinada y dispersa de turismo, como la presencia de un amplio contingente de jubilados con gran capacidad adquisitiva (The Economist, 1999), el incremento del periodo vacacional, la rapidez de los transportes, la repulsa hacia los lugares congestionados a la par que se exige una atención personalizada, y una sensibilidad cada vez mayor por los temas culturales frente a la hegemonía tradicional de los puramente recreativos. Estos últimos ya no se ofrecen como un paquete cerrado, sino que se acompañan con un abanico de visitas a museos o de participaciones en actos culturales y folclóricos.

Según Cooper (1993) existen varios factores para medir el impacto cultural producido por el turismo sobre una pequeña comunidad: el volumen de visitantes, el tipo de economía local, el modelo turístico implantado, la fragilidad del medio y las diferencias socioculturales entre ambos grupos. La repercusión de una política turística inadecuada sobre una población trádicional desprotegida puede acabar destruyendo sus señas de identidad. La población autóctona es un valor añadido irreemplazable dentro de la oferta turística, que precisa de una planificación minuciosa para preservar el equilibrio del medio, sobre todo desde el punto de vista cultural (Taylor, 1995).

Finalmente, aun cuando estamos hablando del impacto cultural del turismo, está claro que éste es difícilmente deslindable del impacto económico, tanto entre los visitantes como entre la población autóctona. Una vez de vuelta a sus países, los turistas incorporan algunas costumbres en su alimentación y forma de vestir que se traducen en demanda de productos de los lugares visitados. Y a la inversa, éstos se ven impelidos a la importación de tecnología más avanzada para atender en viajes próximos las exigencias de sus huéspedes.

Asi pues, el turismo fomenta el progreso y las relaciones culturales en ambos sentidos. Pero sobre todo rompe el aislamiento y rebaja el exceso 
de autoestima a que tiende toda cultura. Existen algunos riesgos para las culturas más débiles al entrar en contacto con otras económicamente más poderosas, aunque ésta es una situación inevitable en un mundo sin fronteras y en el que el turista avanza victorioso enarbolando el dólar y, en breve, también el euro.

\section{BIBLIOGRAFIA}

BARKIN, D. (1996) "Ecoturism: A Tool for Sustainable Development». (Documento de Internet disponible en http://www.greenbuilder.com)

Blazquez, F. (1997) Diccionario de las Ciencias Humanas. Estella: Verbo Divino.

Bote Gómez, V. (1990) Planificación Económica del Turismo. México: Trillas.

COOPER, C. ET AL. (1993) Tourism Principles and Practice. London: Longman.

Cuadrado Roura, J. R. y A. Arranz Calvo (1996) "Los impactos económicos del turismo desde la perspectiva del análisis input-output" en A. PEDREÑo MuÑoz (dir.) Introducción a la Economía del Turismo en España. Madrid: Civitas. págs. 181-216.

Diaz Álvarez, A. (1996) "Turismo y medio ambiente: Análisis económico" en A. Pedreño MUÑOZ (dir.) Introducción a la Economía del Turismo en España. Madrid: Civitas. págs. 275293.

Esomar (1998). Standard Demographic Classification. Amsterdam: ESOMAR.

FIgUeROLA PALOMO, M. (1999a) “Contribución de la actividad turística a la economía española" en V. BOTE GÓmez (dir.) La Actividad Turística Española en 1997. Madrid: O.M.T.

figuerola Palomo, M. (1999b) "El turismo y el sistema económico español» en M. Bayón MARINE (dir.) 50 Años del Turismo Español. Madrid: Centro de Estudios Ramón Areces. págs. 281-301.

Francesscutti, P. (1997) "Turismo en la aldea global» en M. Toharia (dir.) El Futuro que viene. Madrid: Temas de Hoy. págs. 425-435.

Franco Sala, L. (1995) Política Económica del Medio Ambiente. Barcelona: Cedecs.

IOANnides, D. y K. G. DebBage (1998) The Economic Geography of the Tourist Industry. London: Routledge.

JimÉnEZ MORENo, F. J. (1997) Apuntes de Introducción al Turismo. Ávila: fundación Cultural Santa Teresa.

MOREY I ANDREU, M. (1991) "L'impacte del tourisme sobre el medi ambient" en M. MOREY I ANDREU (dir.) Turisme i Medi Ambient a Les Illes Balears. Mallorca: El Tall.

Organización Mundial del TuRismo (1993) Desarrollo Turístico Sostenible. Madrid: O.M.T.

Organización Mundial del TuRISmo (1999) Compendio de Estadísticas del Turismo. 1993-1997. Madrid: O.M.T.

PearCE, D. (1989) Tourist Development. Essex: Longman.

RUESCA, S. y G. DuRAN (1995) Empresa y Medio Ambiente. Madrid: Pirámide.

TAYLOR, G. (1995) 'The Community Apágsroach: Does it really work?' in Tourism Management, vol. $16, n^{\circ} .7$.

THE ECONOMIST (1999) 'Ageing Working' in The Economist, 4 de septiembre.

Vera Rebollo, J. F. (1992) «La dimensión ambiental de la planificación turística: Una nueva cultura para el consumo turístico" en Papers de Turisme, 10: 23-39.

Vera Rebollo, J. F. (1997) Análisis Territorial del Turismo. Barcelona: Ariel.

VERA REBOLLO, J. F. y M. MARCHENA GÓMEZ (1996) «El modelo turistico español: Perspectiva económica y territorial" en A. PEDREÑO MUÑOZ (dir.) Introducción a la Economía del Turismo en España. Madrid: Civitas. págs. 327-364.

Worlo TOURISM ORGanisation (1993) Tourism to the Year 2000. Madrid: W.T.O. 